\title{
Quantitative Study of Electric Conduction in Mineral Oil by Time Domain and Frequency Domain Measurement
}

\author{
Yuan Zhou, Miao Hao, George Chen \\ Electronics and Electrical Engineering \\ University of Southampton \\ SO17 1BJ, UK \\ Gordon Wilson and Paul Jarman \\ National Grid UK \\ CV34 6DA, UK
}

\begin{abstract}
Polarization and depolarization current measurement is a non-destructive testing method to study the dielectric properties of insulating materials. Two types of mineral oils with different aging times have been studied using both time domain and frequency domain methods separately. In this paper, a novel approach to understanding the correlation between the electric conduction in time domain and frequency domain has been proposed. When the charge carriers drift to the electrodes, they may be blocked and accumulated near the electrode. The amount of charge in the vicinity of electrode has been estimated. In the depolarization period, these charge layers will return to the bulk resulting in a depolarization current. In this paper, an analytical solution to the time dependence of the depolarization current has been developed and used to fit the experimental results. The field and temperature dependence of the polarization and depolarization current have been used to study the characteristics of charge carriers in mineral oil. The calculated conductivity has been used to simulate the frequency response in mineral oil and the simulating results have been compared with the experimental data.
\end{abstract}

Index Terms - Current, conductivity, mineral oil, electric field, diffusion.

\section{INTRODUCTION}

MINERAL oil, as a non-conducting insulation and a good coolant, has been widely used in high voltage equipment. The degradation of mineral oil is recognized as one of the major causes of the failure of high voltage equipment [1-2]. Thus, accurate evaluation of dielectric properties of mineral oil can surely benefit the power industry. Polarization and depolarization current technique has been employed in the assessment of insulating status. It has been accepted that moisture, ageing, temperature and electric field can affect the PDC characteristics of insulating media [3-5].

In 1933, Jaffé proposed a polarization theory in crystals under the assumption that the two metal electrodes are completely blocked [6]. This theory has been extended in other media and confirmed experimentally [7-9]. In Jaffé's work, a series of exponential expressions has been suggested to describe the polarization and depolarization process in liquid [9]. Later, Macdonald pointed out that Jaffé's assumption that the density of

Manuscript received on 12 November 2014, in final form 18 May 2015, accepted 12 June 2015. the charge carriers did not change much during the polarization and depolarization process was only valid under a voltage of 0.2 $\mathrm{V}$, which was much lower than the voltage applied in Jaffe's experiment [10]. The classic ionic drift and diffusion model has been widely employed in the study of the electric conduction in mineral oil [11-13]. Gafvert et al have studied the field distortion between two parallel electrodes under DC field [12]. The ionic mobility has been estimated using the ionic drift and diffusion model by many researchers [11-13]. Recently, Lavesson et al proposed a computer based method to simulate the field dependence of electric conduction and they found that the simulation results had a good agreement with the experimental results [13]. However, how to evaluate the high field resistivity of mineral oil is still under debate and this topic has been discussed in CIGRE Study Committee A2: joint working group JWG A2/D1.41 "Transformer insulation - oil conductivity".

Although a lot of researches on polarization and depolarization in mineral oil have been carried out, it is still not clear how to relate the time domain experimental results to the frequency domain results through using the ionic drift and diffusion theory. Besides, the correlation between the motion of the charge carriers and the polarization and depolarization current has not been well 
understood. In our previous work, we have found that the AC conductivity increases with ageing period, whilst the frequency dependent permittivity does not change much within a frequency range from 100 to $0.01 \mathrm{~Hz}$ [14]. With Frood's assumption that there might be high mobility and low density charge carriers in dielectric liquid, this frequency domain dielectric behavior can be explained [15]. Later, a space charge polarization theory based on this assumption has been developed [14]. As the mechanism of the electric conduction in mineral oil must be consistent under both AC and DC field, the polarization theory that was developed in frequency domain should also be valid under DC field.

In this paper, the electric conduction of two kinds of mineral oils with different aging times will be measured using the polarization and depolarization current techniques. An analytical theory of ionic conduction will be developed. The parameters used in the calculation will be employed to calculate the real part of the complex permittivity. The calculated results will be compared with the experimental data. Analysis and interpretations of the experimental results will also be present in this paper.

\section{THEORY OF ELECTRIC CONDUCTION IN MINERAL OIL IN TIME DOMAIN}

If Frood's assumption of the existence of the high mobility charge carriers in dielectric liquid is valid [15], when the mineral oil is depolarized, all these fast charge carriers will be extracted in a very short of time. Thus, the depolarization current mainly relies on the motion of the ionic charge carriers. As the space charge polarization theory [14], which is based on this assumption, indicates that the total conductivity is only partially contributed by the motion of the ions, the conductivity calculated from time domain experimental results should also be lower than the experimental value. In this chapter, an analytical expression to estimate the conductivity from the depolarization current will be proposed.

\subsection{POLARIZATION CURRENT AND ADSORPTION OF IONS}

Let us consider a pair of parallel plate metal electrodes filled with mineral oil. The distance between the two electrodes is $l$. Assuming that the mineral oil can be treated as a liquid, in which the density of free ions is far smaller compared to that of the ionic pairs, the dissociation and recombination process can be described as

$$
\frac{d n_{+}}{d t}=\frac{d n_{-}}{d t}=K_{d} c-K_{r} n_{+} n_{-}
$$

where, $c$ is density of ionic pairs, $n_{+}$and $n_{-}$is density of positive and negative ions in the mineral oil, $K_{d}$ is the dissociation rate and $K_{r}$ is the recombination rate.

If cations and anions are distributed evenly between the two metal electrodes and the diffusion coefficient, the mobility, the density and the charge that is carried by a single charge carrier of positive ions are equal to those of negative ions, the density of positive and negative ions can be denoted as

$$
n_{0}=\frac{\sigma}{q \times\left(\mu_{+}+\mu_{-}\right)}
$$

where, $n_{0}$ is the initial density of positive or negative ions, $\sigma$ is the conductivity of mineral oil, $\mu_{+}$and $\mu_{-}$are the mobility of the positive and negative ions, respectively, and $q$ is the charge carried by a single charge carrier.

The recombination rate can be calculated using Langevin approximation,

$$
K_{r}=\frac{q \times\left(\mu_{+}+\mu_{-}\right)}{\varepsilon_{0} \varepsilon_{r}}
$$

in which, $\varepsilon_{0}$ is the permittivity of vacuum and $\varepsilon_{r}$ is the relative permittivity of mineral oil.

According to Onsager's field enhanced dissociation theory, the dissociation rate can be written as [16]

$$
K_{d}=K_{d}^{0} \frac{I_{1}(4 b)}{2 b}
$$

with

$$
b=\sqrt{\frac{q^{3} E}{16 \pi \varepsilon_{0} \varepsilon_{r} k^{2} T^{2}}}
$$

where, $I_{1}$ is the modified Bessel function of the first kind, $K_{d}^{0}$ is the dissociation rate in the absence of electric field, $E$ is the electric field strength, $k$ is the Boltzmann constant and $T$ is the absolute temperature.

In equilibrium state, the dissociation rate in the absence of electric field can be denoted as

$$
K_{d}^{0}=K_{r} n_{0}^{2} / c
$$

Here, the electric conduction will be discussed under a medium electric field strength in which the newly generated charge carriers can be extracted from the bulk within one second without introducing significant parasitic effects, such as, electrohydrodynamic motion, charge injection and charge convection. In this paper, the electric conduction will be discussed under the field strength ranges from $1 \mathrm{kV} / \mathrm{mm}$ to 5 $\mathrm{kV} / \mathrm{mm}$. In this regime, the recombination of free ions can be neglected and the current is mainly contributed by the motion of the newly generated charge carriers. As indicated by Pontiga and Castellanos, if the internal field can be approximated by the mean electric field, the current density induced from ionic dissociation, $j_{d i s}$, can be written as [17-18]

$$
j_{\text {dis }} \approx 2 K_{d} c l q
$$

On considering a situation that an electric potential of $1 \mathrm{kV}$ is applied on a liquid sample with thickness of $1 \mathrm{~mm}$, the ionic charge carriers with a mobility of $1 \times 10^{-5} \mathrm{~cm}^{2} /(\mathrm{s} \cdot \mathrm{V})$ can travel a distance of $1 \mathrm{~mm}$ in one second. Therefore, all the pre-existing charge carriers in the liquid sample can reach the electrode within one second. For a completely blocked electrode, the charge in the vicinity of one electrode can be estimated using

$$
\rho_{d}=\frac{1}{2}\left[S l \sigma_{d} /\left(2 \mu_{d}\right)+2 K_{d}{\text { clq } S t_{p}}_{p}\right]
$$

in which, $S$ is the surface area of the metal electrode, $\sigma_{d}$ is the conductivity contributed by the motion of the charge carriers 
dissociated from ionic pairs, $\mu_{d}=\mu_{+}=\mu_{-}$is the mobility of the ionic charge carriers and $t_{p}$ is the electrification time.

The boundary conditions have been discussed previously by Jaffé et al [7-9]. Assuming that there exists a state of equilibrium concentration at the electrodes in the absence of an applied voltage and the rate of discharge of ions is proportional to the difference between the instantaneous values and the equilibrium value, for the positive ions, the current density at the electrode can be denoted as [9]

$$
\begin{cases}j_{\text {dis }}(0, t)=-\xi q\left(n_{+}(0, t)-n_{+}{ }^{*}\right) & n_{+}(0, t) \geq n_{+}{ }^{*} \\ j_{\text {dis }}(0, t)=0 & n_{+}(0, t)<n_{+}{ }^{*} \\ j_{\text {dis }}(l, t)=\xi q\left(n_{+}(l, t)-n_{+}{ }^{*}\right) & n_{+}(l, t) \geq n_{+}{ }^{*} \\ j_{\text {dis }}(l, t)=0 & n_{+}(l, t)<n_{+}{ }^{*}\end{cases}
$$

where, $\xi$ is the adsorption coefficient and $n_{+}{ }^{*}$ is the equilibrium value for the density of the positive ions which depends on the nature of the liquid. If we assume the distribution of the ions is homogeneous between the two parallel electrodes, $n_{+}{ }^{*}=n_{0}$.

Assuming that most of the ionic charge carriers will be trapped in a small region that is close to the electrode and these charge carriers will be distributed evenly in that region, the total charge in the charge layer obeys the following expression,

$$
\frac{d \rho_{d}}{d t} \approx K_{d} c S l-\xi\left(n_{+}-n_{0}\right) \Delta V=K_{d} c S l-\xi\left(\rho_{d}-n_{0} \Delta V\right)
$$

where $\Delta V$ is the volume of a region close to the electrode and $\rho_{d}$ is the amount of charge in the charge layer. The first term on the right side represents the newly generated ionic charge carriers; the second term describes the process that the ionic charge carriers are neutralized by the electrode.

On considering that most of the initially dissociated ionic charge carriers are moved to a close region of the electrode within one second and only the long-term conductivity are interesting here, $\rho_{d}$ at time $t=0$ can be given in the form of

$$
\rho_{d}(0)=\frac{\sigma_{d} S l}{4 \mu_{d} q}
$$

The solution of equation (10) is represented by

$$
\begin{aligned}
p(d, t)= & \left(\frac{\sigma_{d} S l}{4 \mu_{d} q}-\frac{K_{d} c S l+\xi n_{0} \Delta V}{\xi}\right) e^{-\xi t} \\
& +\frac{K_{d} c S l+\xi n_{0} \Delta V}{\xi}
\end{aligned}
$$

As seen from equation (12), $\xi$ is also a time constant which indicates the charging time of the charge layer. However, there is no report concerning this parameter in mineral oil.

In an equilibrium state, the amount of charge in a charge layer can be estimated using the following equation,

$$
\rho(\infty)=\frac{I_{1}(4 b)}{2 b \xi} K_{r} n^{2} S l+n_{0} \Delta V
$$

\subsection{DEPOLARIZATION CURRENT}

If the electric field can be treated to be homogeneous, the depolarization will be only affected by the diffusion of the ionic charge carriers. The assumptions that all charge carriers can be blocked by the electrode will be made here. As these charge carriers cannot get through the electrodes, they can only move backwards into the bulk. If the diffusion only happens in 1-D system and there are only positive charge carriers in the mineral oil, the current density at point $x$ can be denoted as,

$$
j_{d e p}(x, t)=q D_{+} \frac{d n_{+}}{d x}
$$

where, $j_{\text {dep }}(x, t)$ is the depolarization current density and $D_{+}$is the diffusion coefficient.

With conservation of mass, the dependence of charge density upon the time can be denoted as

$$
D_{+} \frac{\partial^{2} n_{+}}{\partial x^{2}}=\frac{\partial n_{+}}{\partial t}
$$

When the DC field is removed, these charge carriers will diffuse back into the bulk. Due to the fact that the distribution of the charge carriers in the charge layer is not very clear, it would be unpractical to calculate the diffusion current. Here, an image method is going to be proposed so that the diffusion current can be calculated without the knowledge about the charge distribution in the charge layer. The scheme of this method is illustrated in figure 1. If the ionic diffusion in the middle of the bulk can be ignored, the boundary condition can be written as

$$
n_{+}(0, t)=n_{+}(l, t)=0
$$

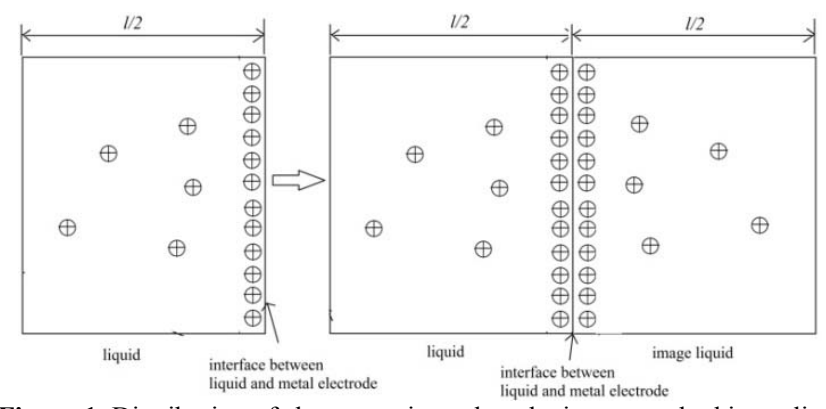

Figure 1. Distribution of charge carriers when the image method is applied.

The solution to equations (14) - (16) is similar to that of 1-D thermal diffusion equations [19]. It is easy to obtain the general solution by direct substitution. The charge distribution at the moment that the external field is removed can be described as

$$
n_{+}(x, 0)=f(x)
$$

Therefore, the solution to equation (14) can be denoted as

$$
n_{+}(x, t)=\sum_{s=1}^{\infty} B_{s} \sin \left(\frac{s \pi}{l} x\right) \exp \left[-\left(\frac{s \pi}{l}\right)^{2} D_{+} t\right]
$$

in which, $B_{s}$ is the coefficient related to the initial charge distribution and $s$ is a positive integer.

Employing the initial condition of equation (17), the coefficient $B_{s}$ can be calculated using the following 
expression,

$$
B_{s}=\frac{2}{l} \int_{0}^{l} f(x) \sin \left(\frac{s \pi}{l} x\right) d x
$$

On considering the symmetry of the charge distribution in figure 1 , the expression for the charge distribution can be denoted as

$$
Q(x, t)=q \times n_{+}(x, t)=\sum_{s=1}^{\infty} q \times B_{S} \times u_{S}(x, t)
$$

with

$$
\left\{\begin{array}{c}
u_{s}(x, t)=\exp \left(-\left((2 s-1) \frac{\pi}{l}\right)^{2} D_{+} t\right) \sin \left((2 s-1) \frac{\pi}{l} x\right) \\
B_{s}=\frac{2}{l} \int_{0}^{l} f(x) \sin \left((2 s-1) \frac{\pi}{l} x\right) d x
\end{array}\right.
$$

where $Q(x, t)$ is the charge density. As we are not interested in the current that is measured right after the sample is shortcircuited, those high order components can be ignored, thus, the charge density can be written as

$$
Q(x, t) \approx\left(B_{1} q\right) \exp \left(-\frac{\pi^{2} D}{l^{2}} t\right) \sin \left(\frac{\pi}{l} x\right)
$$

If the total charge in one charge layer is $Q_{1}, B_{1}$ can be approximated as

$$
B_{1} \approx 4 Q_{1} /(l q)
$$

As seen from equation (20b), if the thickness of the charge layer is much shorter than the length between the two metal electrodes, $\sin \left((2 s-1) \frac{\pi}{l} x\right) \approx 1$. Thus, if the image method is employed, the depolarization current can be calculated without the knowledge of the distribution of charge carriers in the charge layer.

With conservation of electric charge

$$
\frac{\partial Q(x, t)}{\partial t}=\frac{\partial j_{d e p}(x, t)}{\partial x}
$$

The depolarization current density can be denoted as

$$
j_{d e p}(x, t)=\frac{4 \pi D_{+} Q_{1}}{l^{2}} \exp \left(-\frac{\pi^{2} D_{+}}{l^{2}} t\right)\left(\cos \left(\frac{\pi}{l} x\right)-1\right)
$$

As the current between the two metal electrodes are not homogeneous, thus, an integration will be employed here to calculate the current flowing through the measuring circuit. The total current density that flows in the external circuit, $J_{+}(t)$, can be calculated as

$$
J_{+}(t)=\frac{2}{l} \int_{0}^{l / 2} j_{d}(x, t) d x=-\frac{4(\pi-2) Q_{1} D_{+}}{l^{2}} \exp \left(-\frac{\pi^{2} D_{+}}{l^{2}} t\right)
$$

If there are multiple kinds of charge carriers, this current density can be written as

$$
J_{+}(t)=-\sum_{a=1}^{n} \frac{4(\pi-2) Q_{a} D_{a}}{l^{2}} \exp \left(-\frac{\pi^{2} D_{a}}{l^{2}} t\right)
$$

in which, $Q_{a}$ and $D_{a}$ are the total charge in the charge layer and the diffusion coefficient for the $a$-th charge carriers.

For negative charge carriers, we can obtain

$$
J_{-}(t)=\sum_{a=1}^{n} \frac{4(\pi-2) Q_{a} D_{a}}{l^{2}} \exp \left(-\frac{\pi^{2} D_{a}}{l^{2}} t\right)
$$

Here, we assume that the diffusion coefficient, mobility and number density of positive ions are equal to those of negative ions. The total depolarization current density, $J_{t o t}(t)$, that is contributed by both positive and negative charge carriers can be written as

$$
J_{\text {tot }}(t)=J_{+}(t)+J_{-}(t)=-\sum_{a=1}^{n} \frac{8(\pi-2)\left|Q_{a}\right| D_{a}}{l^{2}} \exp \left(-\frac{\pi^{2} D_{a}}{l^{2}} t\right)(28)
$$

As seen from equation (28), the exponential terms are similar to that derived by Jaffé and Adamczewski $[9,20]$. How to use equation (28) to calculate the DC conductivity will be discussed later.

\section{EXPERIMENTAL SETUP}

The electrode system that was designed by Küchler has been used in the polarization and depolarization current measurement [21]. This electrode system was placed in a sealed glass test cell. The distance between the high voltage electrode and the measuring electrode is $1 \mathrm{~mm}$, the gap between the measuring electrode and guard electrode is 0.5 $\mathrm{mm}$ and the edge radiuses of the measuring electrode and the guard electrode are $0.5 \mathrm{~mm}$. The test cell was evacuated first and then injected with the mineral oil from a vacuum vessel to avoid contamination from the air. The tests were performed under different temperatures $\left(30\right.$ and $\left.90{ }^{\circ} \mathrm{C}\right)$ and different electric field strengths $(1,2$ and $5 \mathrm{kV} / \mathrm{mm})$.

Two kinds of mineral oils have been tested, the fresh mineral oil (oil A), and the aged mineral oil was taken from a HVDC converter transformer which has been in service for ten years (oil B). A DC voltage was applied across the test object for three hours. When the voltage was removed, the object was short-circuited and the depolarization current was recorded.

Solartron dielectric interface 1296 and model 1260A impedance/gain-phase analyser have been used for the dielectric spectroscopy measurement. The voltage across the sample was $1 \mathrm{~V}$. The experiment was performed at two different temperatures $\left(30\right.$ and $\left.90^{\circ} \mathrm{C}\right)$ and the distance between the two metal electrodes is $0.5 \mathrm{~mm}$. The frequency range for the test was $100-0.01 \mathrm{~Hz}$.

\section{EXPERIMENTAL RESULTS}

The results are divided into polarization current and depolarization sections.

\subsection{POLARIZATION CURRENT}

Figures 2 and 3 show the time dependence of the polarization current of oil $\mathrm{A}$ and $\mathrm{B}$ under different temperatures and electric field strengths. The blue, red and green lines represent the time dependence of the polarization current of oil A under the electric field strengths of 1000 $\mathrm{V} / \mathrm{mm}, 2000 \mathrm{~V} / \mathrm{mm}$ and $5000 \mathrm{~V} / \mathrm{mm}$, respectively. When the measuring temperature is $30^{\circ} \mathrm{C}$, the steady-state current does not change much with the electric field strength and it seems the polarization current has entered a saturated region and no longer obeys Ohm's law [20]. The initial current seems to increase with the electric field strength at both 30 and $90{ }^{\circ} \mathrm{C}$, which indicates the velocity of the charge carriers can increase 
with the electric field strength. The steady- state current that measured at $90{ }^{\circ} \mathrm{C}$ does not change much when the electric field strength increases from 1000 to $2000 \mathrm{~V} / \mathrm{mm}$, whilst a significant increase can be observed under a field strength of $5000 \mathrm{~V} / \mathrm{mm}$. It suggests that the polarization current of oil A has passed the saturated region and starts to increase again.

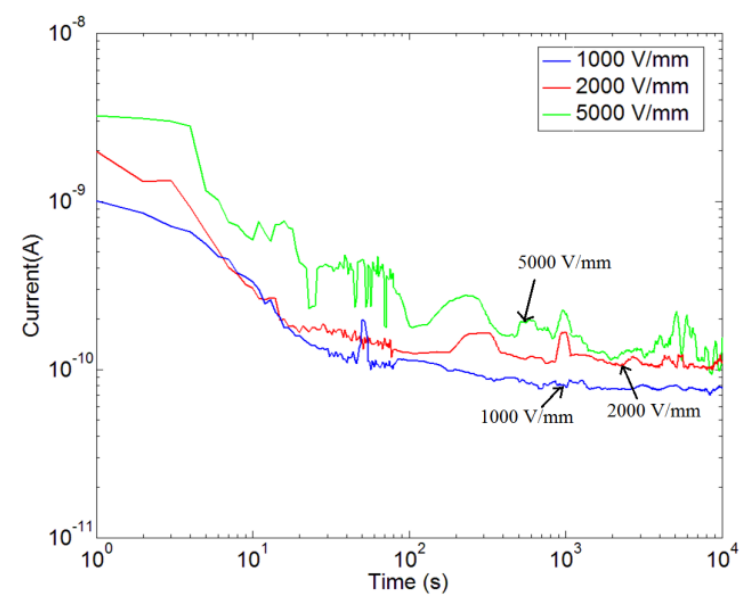

Figure 2. Time dependence of the polarization current of oil A under different electric field strength at $30^{\circ} \mathrm{C}$.

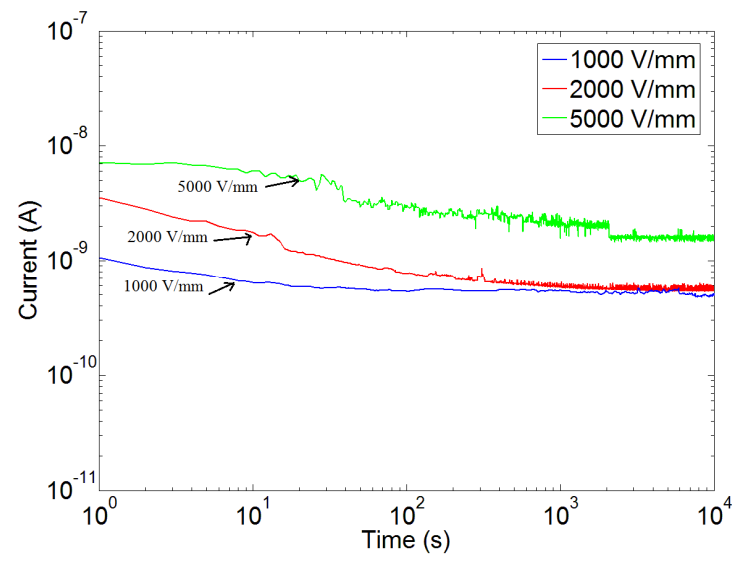

Figure 3. Time dependence of the polarization current of oil A under different electric field strength at $90^{\circ} \mathrm{C}$.

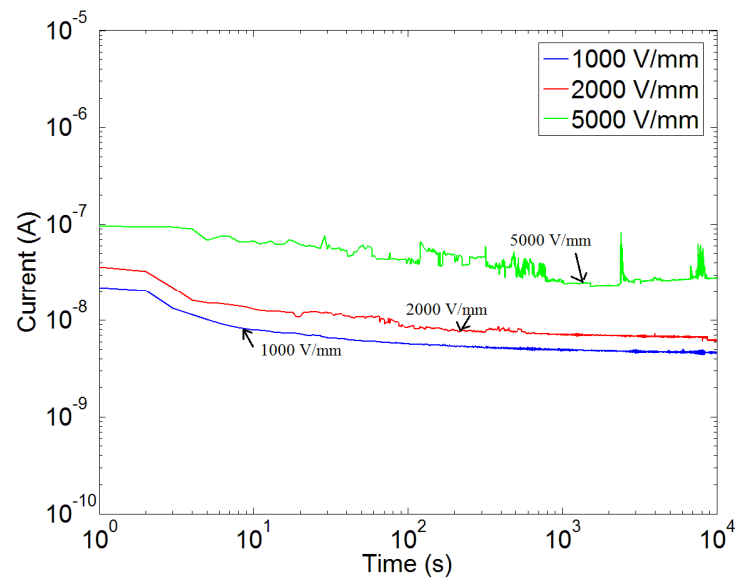

Figure 4. Time dependence of the polarization current of oil B under different electric field strength at $30^{\circ} \mathrm{C}$.

The time dependence of the polarization current of oil B under different fields strengths and temperatures is shown in figures 4 and 5. The initial current and the steady- state current increase with the electric field strength at both 30 and $90^{\circ} \mathrm{C}$. It seems there is no saturated region for the field dependent electric conduction in oil B. In contrast with the experimental results of oil $\mathrm{A}$, the curves of the time dependence of the polarization current are smoother under a field of 1000 and $2000 \mathrm{~V} / \mathrm{mm}$. The measured current becomes unstable when the field strength is $5 \mathrm{kV} / \mathrm{mm}$. It has been reported that under high electric field, instability that are characterised by violent fluctuations in the magnitude of the current can be observed $[20,22]$. Nosseir et al claimed that this fluctuation was caused by the neutralization of the impurities and the charge injection at the electrode [22]. The current also increases with the temperature. The steady- state current of oil B measured at 90 ${ }^{\circ} \mathrm{C}$ is about 10 times higher than that measured at $30^{\circ} \mathrm{C}$.

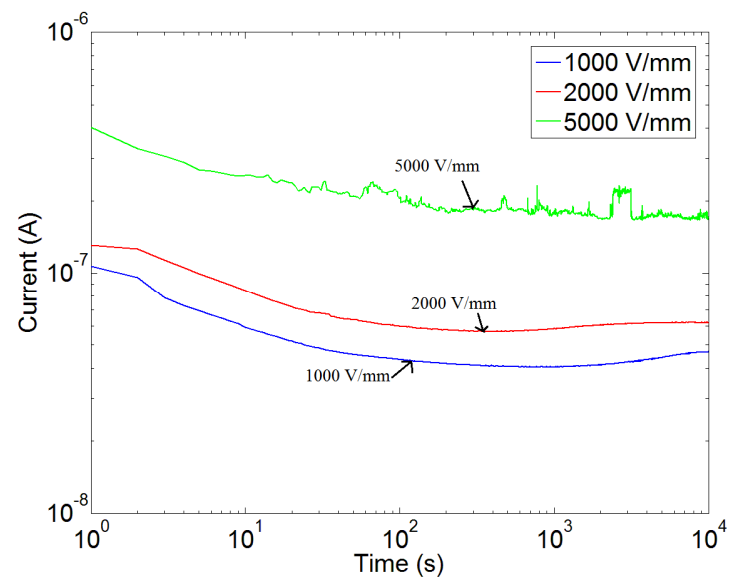

Figure 5. Time dependence of the polarization current of oil B under different electric field strength at $90^{\circ} \mathrm{C}$.

\subsection{DEPOLARIZATION CURRENT}

Figures 6-7 depict the time dependence of the depolarization current of oil A under different field strengths at 30 and $90{ }^{\circ} \mathrm{C}$, respectively. Figures $8-9$ show the time dependence of the depolarization current of oil $\mathrm{B}$ under different temperatures and field strengths. The circular, square and star markers represent the experimental results obtained under the electric field strengths of 1000, 2000 and $5000 \mathrm{~V} / \mathrm{mm}$, respectively.

As seen from figures 6-9, the maximum values of the magnitude of the depolarization current of both oil A and oil $B$ increase with the electric field strength and they decrease faster under a higher electric field strength. It is accepted that the dissociation rate and the ionic injection rate increase with the field strength [16, 23-27], and consequently there should be more charge carriers participating in the electrical conduction process under a higher field strength. As more charge carriers will be blocked by the electrodes, the amount of charge in the charge layer will also increase with the electric field strength. When the oil sample is short-circuited, the charge carriers that are stuck in the vicinity of the electrodes will start to drift backwards to the bulk causing a depolarization current. Thus, the magnitude of the depolarization current should also increase with the field strength. It seems this explanation is consistent with results of the depolarization current measurement. 


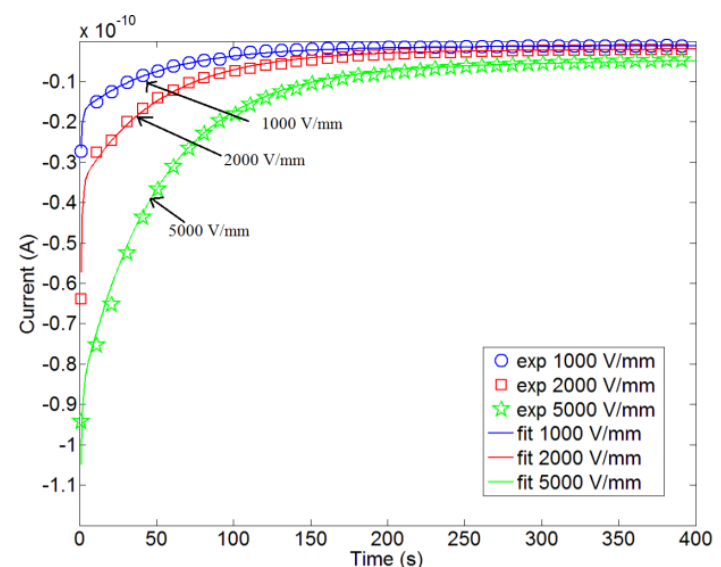

Figure 6. Comparison between the experimental value of the depolarization current of oil A at $30^{\circ} \mathrm{C}$ and the theoretical fits according to equation (29)

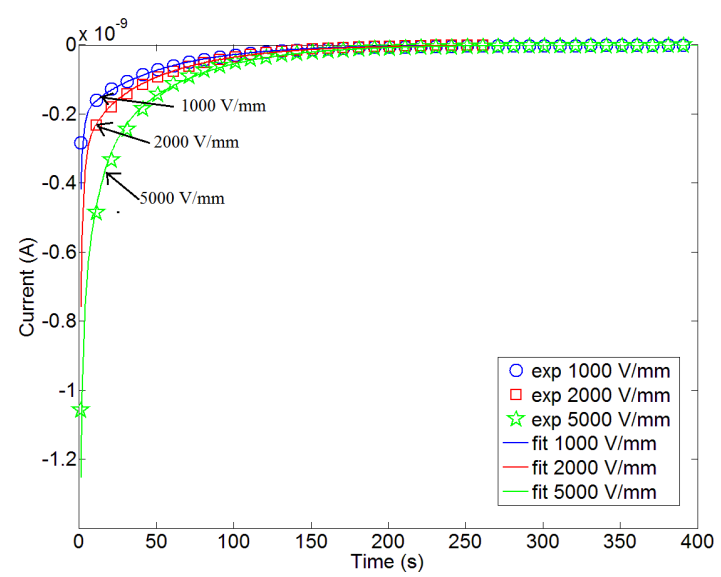

Figure 7. Comparison between the experimental value of the depolarization current of oil A at $90^{\circ} \mathrm{C}$ and the theoretical fits according to equation (29).

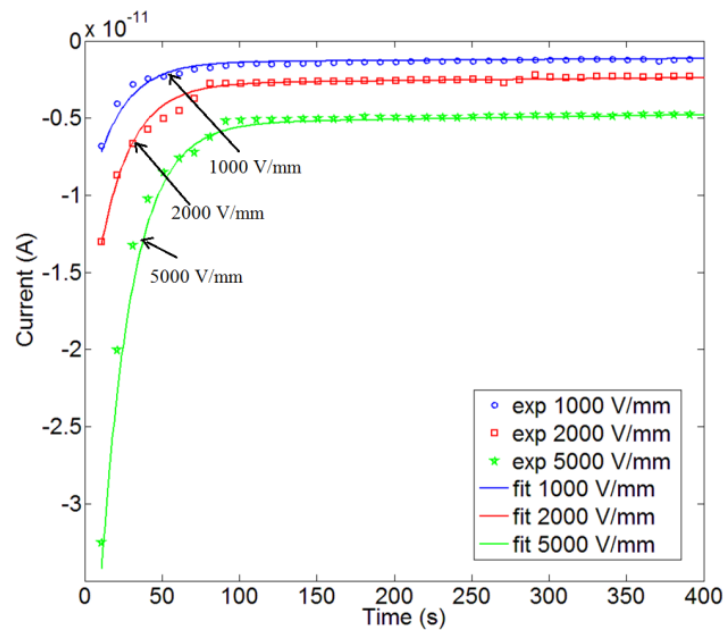

Figure 8. Comparison between the experimental value of the depolarization current of oil B at $30^{\circ} \mathrm{C}$ and the theoretical fits according to equation (29).

As seen from figures 6-9, when the temperature increases, a bigger magnitude of the depolarization current can be observed. The viscosity of the mineral oil decreases with temperature, thus, the friction force due to the viscosity is reduced when the temperature is increased. Besides, the dissociation rate increases with the temperature and more charge carriers are generated at a higher temperature [28]. Consequently, there will be more charge in the charge layer at $90^{\circ} \mathrm{C}$. When the external voltage is removed, these charge carriers in the charge layer will move backwards to the bulk between the two metal electrodes. A higher mobility of the charge carriers can result in a faster depolarization. Therefore, when the test media is depolarized, the magnitude of the depolarization current induced by the drift and diffusion of the charge carriers in the charge layer increases with the temperature. Although the conductivity of oil B is much higher than that of oil A, the magnitudes of the depolarization current of these two oils obtained at the same temperature and electric field are similar, which suggests the electric conduction in mineral oil may not contributed by the ionic charge carriers only.

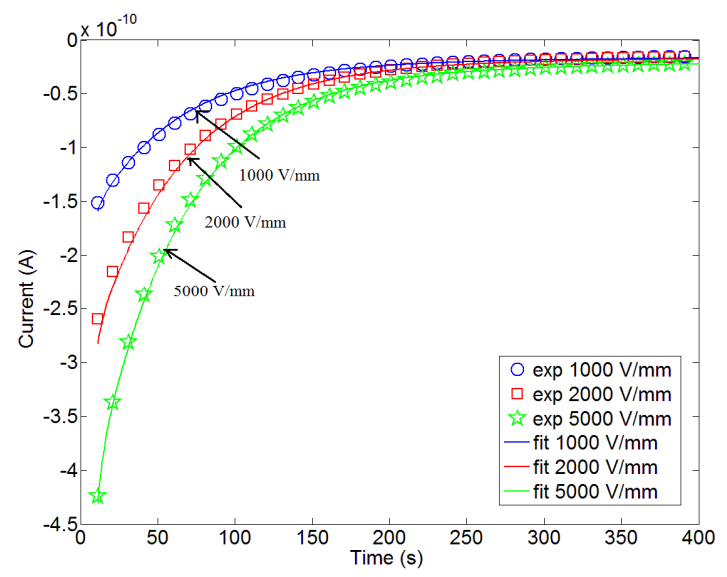

Figure 9. Comparison between the experimental value of the depolarization current of oil B at $90^{\circ} \mathrm{C}$ and the theoretical fits according to equation (29)

\section{DEPOLARIZATION CURRENT AND FREQUENCY DOMAIN DIELECTRIC SPECTROSCOPY}

In this section, how to relate the time domain results with the frequency domain results will be present. The conductivity calculated from the depolarization current results will be substituted by the polarization theory that was developed in frequency domain to obtain the relevant frequency responses. If Frood's assumption is valid [15], the simulated frequency response should be close to the experimental value.

As seen from equation (28), the curve for depolarization current can be calculated by a formula of the type

$$
I_{d e p}=J_{t o t} \times S=-\sum_{a=1}^{n} C_{a} \exp \left(-b_{a} t\right)
$$

As the mineral oil is a mixed compound of paraffin, naphthene and aromatic, there should be charge carriers with different mobility in the mineral oil [1-2]. In this paper, $a$ was set to be 3 to obtain a better fit. The value of parameters $C_{a}$ and $b_{a}(a=1,2,3)$ are obtained from the "try and error" method.

Figures 6-9 show the comparison between theory and measurements on the depolarization current of oil A and oil B. The blue, red and green curves represent the calculated value of the depolarization under field strengths of 1000, 2000 and $5000 \mathrm{~V} / \mathrm{mm}$. The constants used in this calculation for the depolarization current of oil A and oil B are given in Table 1 and Table 2, separately. It has been seen that the theoretical curves can fit the experimental data with good accuracy. 
Table 1. Constants used in the calculation of depolarization current of oil A

\begin{tabular}{c|c|c|c|c|c}
\hline \multirow{2}{*}{ temperature } & \multirow{2}{*}{$a$} & \multirow{2}{*}{$b_{a}$} & \multicolumn{3}{|c}{$C_{a}$} \\
\cline { 4 - 6 } & & & $1 \mathrm{kV} / \mathrm{mm}$ & $2 \mathrm{kV} / \mathrm{mm}$ & $5 \mathrm{kV} / \mathrm{mm}$ \\
\hline \multirow{2}{*}{$30^{\circ} \mathrm{C}}$. & 1 & 1 & $2.5 \times 10^{-11}$ & $6.0 \times 10^{-11}$ & $5.0 \times 10^{-11}$ \\
& 2 & $2 \times 10^{-2}$ & $1.6 \times 10^{-11}$ & $3.3 \times 10^{-11}$ & $8.7 \times 10^{-11}$ \\
& 3 & $1 \times 10^{-3}$ & $1.7 \times 10^{-12}$ & $2.9 \times 10^{-12}$ & $7.2 \times 10^{-12}$ \\
\hline \multirow{2}{*}{$90^{\circ} \mathrm{C}}$. & 1 & $1 \times 10^{-1}$ & $1.9 \times 10^{-10}$ & $1.0 \times 10^{-10}$ & $6.0 \times 10^{-10}$ \\
& 2 & $2 \times 10^{-2}$ & $1.9 \times 10^{-10}$ & $2.6 \times 10^{-10}$ & $3.6 \times 10^{-10}$ \\
& 3 & $2 \times 10^{-3}$ & $2.0 \times 10^{-12}$ & $4.0 \times 10^{-12}$ & $5.0 \times 10^{-12}$ \\
\hline
\end{tabular}

Table 2. Constants used in the calculation of depolarization current of oil B.

\begin{tabular}{c|c|c|c|c|c}
\hline \multirow{2}{*}{ temperature } & \multirow{2}{*}{$a$} & \multirow{2}{*}{$b_{a}$} & \multicolumn{3}{|c}{$C_{a}$} \\
\cline { 4 - 6 } & & & $1 \mathrm{kV} / \mathrm{mm}$ & $2 \mathrm{kV} / \mathrm{mm}$ & $5 \mathrm{kV} / \mathrm{mm}$ \\
\hline \multirow{2}{*}{$30^{\circ} \mathrm{C}}$. & 1 & 1 & $1.5 \times 10^{-11}$ & $4.0 \times 10^{-11}$ & $1.9 \times 10^{-10}$ \\
& 2 & $5 \times 10^{-2}$ & $1.0 \times 10^{-11}$ & $1.8 \times 10^{-11}$ & $5.0 \times 10^{-11}$ \\
& 3 & $4 \times 10^{-4}$ & $1.4 \times 10^{-12}$ & $2.5 \times 10^{-12}$ & $5.4 \times 10^{-12}$ \\
\hline \multirow{2}{*}{$90^{\circ} \mathrm{C}}$. & 1 & $2 \times 10^{-1}$ & $1.5 \times 10^{-10}$ & $3.9 \times 10^{-10}$ & $8.0 \times 10^{-10}$ \\
& 2 & $2 \times 10^{-2}$ & $1.6 \times 10^{-10}$ & $3.0 \times 10^{-10}$ & $4.5 \times 10^{-10}$ \\
& 3 & $7 \times 10^{-4}$ & $2.2 \times 10^{-11}$ & $2.3 \times 10^{-11}$ & $2.9 \times 10^{-11}$ \\
\hline
\end{tabular}

For simplicity, we assume the adsorption coefficient $\xi$ does not change with the electric field. As seen from equation (12), this adsorption coefficient can also be considered as a time constant of polarization. Here, this coefficient is assumed to be subject to the following expression

$$
\xi \approx 1 / \Delta t
$$

where, $\Delta t$ is the settling time that is required for the time domain response to reach the equilibrium state.

The length of the charge layer in the vicinity of the electrode can be estimated by the Debye length of the liquid and the Debye length for non-polar liquid is around several micrometres [29]. As seen from figures 2-5, the settling time to reach the quasi-steady state is higher than $100 \mathrm{~s}$, therefore $\xi<0.01$. The DC conductivity of oil A measured experimentally is higher than $0.10 \mathrm{pS} / \mathrm{m}$. If a Debye length of $10 \mu \mathrm{m}$ is assumed, the following correlation can be easily obtained,

$$
\frac{K_{d} c S l}{\xi n_{0} \Delta V}=\frac{I_{1}(4 b)}{2 b} \frac{2 \sigma_{d} S l}{\varepsilon_{0} \varepsilon_{r} \xi \Delta V}>>1
$$

Therefore, equation (13) can be simplified as,

$$
\rho(\infty) \approx \frac{K_{d} c S l}{\xi}=\frac{I_{1}(4 b)}{2 b \xi} K_{r} n^{2} S l
$$

With equation (28), the charge in the charge layers and the mobility of the charge carriers can be denoted as

$$
\left\{\begin{array}{c}
Q_{a}=\pi^{2} C_{a} /\left[8 S(\pi-2) b_{a}\right] \\
\mu_{a}=b_{a} q l^{2} /\left(k T \pi^{2}\right)
\end{array}\right.
$$

If equations (32) and (33) are compared, the correlation between the conductivity and the total charge in the charge layer can be expressed as

$$
\frac{I_{1}(4 b)}{2 b} K_{r} n^{2} q S l \Delta t=Q_{a} S
$$

The conductivity contributed by the $a$-th charge carriers can be denoted as

$$
\sigma_{d(a)}=2 n \mu_{a} q=\frac{2 b_{a} q^{2} l^{2}}{k T \pi^{2}} \sqrt{\frac{2 Q_{a} b}{q I_{1}(4 b) K_{r} l \Delta t}}
$$

The calculated mobility and the relevant conductivity of oil A and oil B can be found in Tables 3 and 4, respectively. As seen from Tables 3 and 4, the conductivity contributed by the motion of the charge carriers with the same mobility does not change much with the electric field strength, which indicates that the depolarization process may strongly rely on the ionic diffusion. The total calculated conductivity increases with the temperature because of a high dissociation rate and a low viscosity at high temperature. These parameters can provide a brief view of the dielectric characteristics of charge carriers in mineral oil. However, how to relate these calculated values to the physical and chemical properties of mineral oil is still not clear and more research is needed.

Table 3. Calculated conductivity and mobility of charge carriers in oil A

\begin{tabular}{c|c|c|c|c|c}
\hline \multirow{2}{*}{ temperature } & \multirow{2}{*}{$a$} & \multirow{2}{*}{$\mu_{a}\left(\mathrm{~cm}^{2} /(\mathrm{s} \cdot\right.$} & \multicolumn{3}{|c}{$\sigma_{d(a)}(\mathrm{pS} / \mathrm{m})$} \\
\cline { 3 - 6 } & & $\mathrm{V}))$ & $1 \mathrm{kV} / \mathrm{mm}$ & $2 \mathrm{kV} / \mathrm{mm}$ & $5 \mathrm{kV} / \mathrm{mm}$ \\
\hline & 1 & $3.83 \times 10^{-2}$ & 0.38 & 0.49 & 0.27 \\
$30^{\circ} \mathrm{C}$. & 2 & $7.65 \times 10^{-4}$ & 0.31 & 0.36 & 0.36 \\
& 3 & $3.82 \times 10^{-5}$ & 0.10 & 0.11 & 0.10 \\
\hline & 1 & $3.20 \times 10^{-3}$ & 1.36 & 0.82 & 1.21 \\
$90^{\circ} \mathrm{C}$. & 2 & $6.39 \times 10^{-4}$ & 1.36 & 1.31 & 0.94 \\
& 3 & $6.39 \times 10^{-5}$ & 0.14 & 0.16 & 0.11 \\
\hline
\end{tabular}

Table 4. Calculated conductivity and mobility of charge carriers in oil B

\begin{tabular}{c|c|c|c|c|c}
\hline \multirow{2}{*}{ temperature } & \multirow{2}{*}{$a$} & \multirow{2}{*}{$\mu_{a}\left(\mathrm{~cm}^{2} /(\mathrm{s} \cdot\right.$} & \multicolumn{3}{|c}{$\sigma_{d(a)}(\mathrm{pS} / \mathrm{m})$} \\
\cline { 3 - 6 } & & $\mathrm{V}))$ & $1 \mathrm{kV} / \mathrm{mm}$ & $2 \mathrm{kV} / \mathrm{mm}$ & $5 \mathrm{kV} / \mathrm{mm}$ \\
\hline & 1 & $3.83 \times 10^{-2}$ & 0.42 & 0.56 & 0.79 \\
$30{ }^{\circ} \mathrm{C}$. & 2 & $1.92 \times 10^{-3}$ & 0.34 & 0.38 & 0.38 \\
& 3 & $1.53 \times 10^{-5}$ & 0.16 & 0.14 & 0.12 \\
\hline & 1 & $6.39 \times 10^{-3}$ & 1.73 & 2.27 & 1.98 \\
$90^{\circ} \mathrm{C}$. & 2 & $6.39 \times 10^{-4}$ & 1.77 & 1.98 & 1.49 \\
& 3 & $2.24 \times 10^{-5}$ & 0.66 & 0.55 & 0.38 \\
\hline
\end{tabular}

Oil B has been aged for 10 years and suffered from electric, thermal and chemical stresses, therefore, the amount of the ionic charge carriers in oil B should be higher than that in oil A. If the total calculated conductivities that are contributed by the motion of the ionic charge carriers of oil A and oil B are compared, the total conductivity induced by the ionic charge carriers of oil B is slightly higher than that in oil A. However, both the steady- state and the initial conductivity current of oil B experimentally measured are about 30-100 times higher than that of oil A. Thus, other types of charge transportation should be involved in the polarization period. In our previous work, a polarization theory involving the motion of the charge carriers that have low density and high mobility has been proposed. If these high mobility charge carriers do exist, the total conduction should contain two parts: the ionic conduction induced 
from the motion of the ionic charge carriers and the conduction that is induced from those high mobility charge carriers [14]. It has been reported that the ratio of the conductivity contributed by the motion of the ionic charge carriers over the total conductivity decreases with the ageing period [14]. The ionic conductivity of oil A is close to its DC conductivity, which means the majority charge carrier in oil A is the ionic charge carriers. As seen from Table 3 and 4, oil A and oil B have similar ionic conductivity. Considering oil $\mathrm{B}$ has a much higher DC conductivity, the ratio of the ionic conductivity over the total DC conductivity of oil B is lower than that of oil A. Thus, the electric conduction in oil B mainly depends on the motion of the high mobility charge carriers, which is consistent with our previous study in frequency domain. To sum up, the major conduction in fresh oil is the ionic conduction, whilst the high mobility charge carriers conduction is the dominate conduction in aged oil, which has a good agreement with our previous study in the frequency domain measurement [14].

If the velocity of these charge carriers are proportional to the electric field, the motion of these fast charge carriers can only contribute to the imaginary part of the complex permittivity under a homogeneous field [30]. A modified Coelho model has been proposed to explain the dielectric behaviour of mineral oil in frequency domain. This modified model can be denoted as [30]

$$
\varepsilon(\omega)=\varepsilon_{0} \varepsilon_{r}\left[\frac{1+i \omega \tau^{\prime}}{i \omega \tau^{\prime}+\tanh \left(k_{\omega}^{\prime} d\right) / k_{\omega}^{\prime} d}\right]-i \frac{\sigma_{i}}{\omega}
$$

with

$$
\left\{\begin{array}{l}
\tau^{\prime}=\varepsilon_{0} \varepsilon_{r} /\left(\sigma-\sigma_{i}\right) \\
k_{\omega}^{\prime 2}=\frac{1}{D \tau^{\prime}}\left(1+i \omega \tau^{\prime}\right)
\end{array}\right.
$$

where, $\varepsilon_{r}$ is the relative permittivity of the sample, $\omega$ is the angular frequency, $D$ is the diffusion coefficient, $2 d$ is the distance between the two parallel electrodes and $\sigma_{i}$ is the conductivity contributed by the motion of the fast charge carriers. As seen from equation (36), the motion of these fast charge carriers can only affect the imaginary part of the complex permittivity and the real part of the complex permittivity mainly depends on the drift and diffusion of the ionic charge carriers. Since the total conductivity contributed by the ions calculated using equation (35) of oil $\mathrm{A}$ and oil B is close to each other, the real part of the complex permittivity of oil A and oil B should not differ much from each other.

Here, the real part of the complex permittivity of oil A and oil B has been calculated using equation (36) and the calculated values were compared with the data that obtained in experiment. As high electric field strength introduces parasitic phenomena, like Electro hydro dynamic motion, charge injection, charge convection et al, in order to reduce these parasitic effects, only the conductivity under a field strength of $1 \mathrm{kV} / \mathrm{mm}$ was used in this calculation.

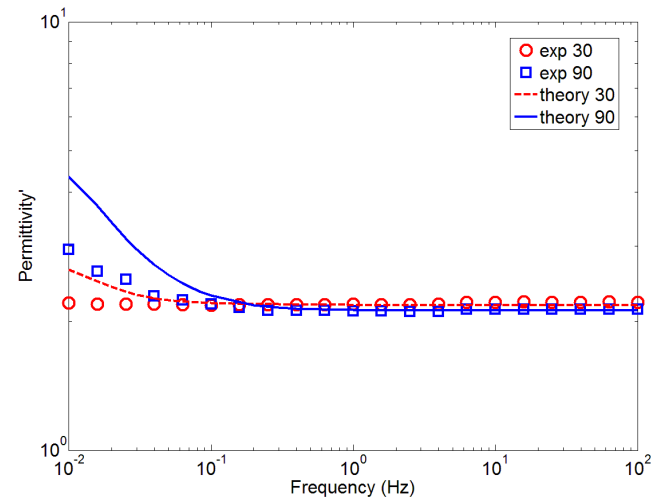

Figure 10. Comparison between theoretical and experimental value of the real part of the complex permittivity of oil A.

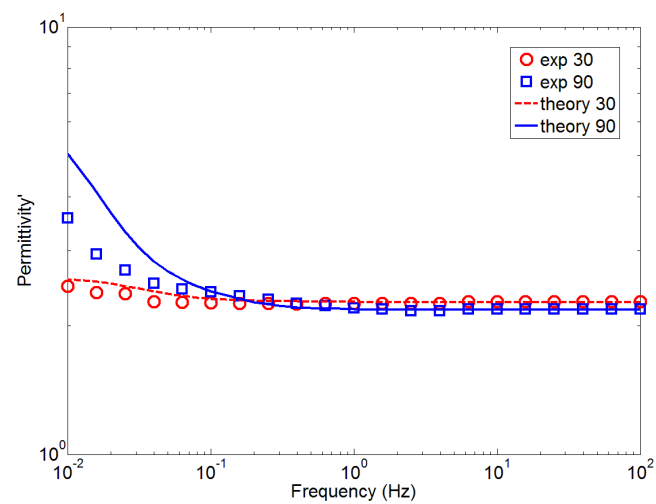

Figure 11. Comparison between theoretical and experimental value of the real part of the complex permittivity of oil B

The calculated values of the real part of the complex permittivity and the values measured experimentally of oil A and oil B are illustrated in figure 10 and 11, respectively. The difference between the experimental values of the real part of the complex permittivity of these two kinds of mineral oil is not significant, which means the space charge polarizations in oil A and oil B can be similar. The calculated complex permittivity using equation (36) is always higher than the experimental data. The calculated plots do not differ much from each other at the same temperature, which is consistent with the experimental results.

As Coelho's model does not take account of the ionic adsorption at the electrode, the real part of the complex permittivity calculated using Coelho's model should be higher than the actual value. Besides, equation (35) used to calculate the dissociated conductivity is not right in a rigid way. As the knowledge about the distribution of the charge carriers in the vicinity of the electrode is still limited and the non-linearity of the ionic drift and diffusion model, to obtain an analytical solution for the depolarization current with the field distortion being taken into account is not practical and equation (35) is only an approximation. If the electric field between the gap of the parallel electrodes is not homogeneous, the charge drift cannot be ignored and the current induced from ionic drift should also be added to the total depolarization current. Therefore, the conductivity calculated using equation (35) is higher than its actual value. If the field is low, equation (32) is not valid as the newly generated charge carriers are unable to reach the electrode in a short of time. When the field is high, 
there are both injected charge carriers and dissociated charge carriers in the mineral oil. These injected charge carriers might react with the dissociated charge carriers or the neutral molecules and generate new charge carriers. Therefore, the analysis method proposed in this paper is only valid for a medium electric field. Also, the coefficient of the extraction at the electrode cannot be measured directly by the experiment and this coefficient may change with the electric field. When the field is high, the measured experimental results are fluctuated due to the non-linearity in the mineral oil and to acquire this coefficient through the method proposed in this paper becomes difficult.

\section{CONCLUSION}

The conductivity of the mineral oil increases with ageing and temperature. At high electric field, the conducting current becomes unstable and violent fluctuation can be observed. The permittivity of the mineral oil does not change within the frequency range from 100 to $1 \mathrm{~Hz}$. If the frequency has a further decrease from $1 \mathrm{~Hz}$, the permittivity of the mineral oil increases. Although the difference between the conductivity of these two types of mineral oils are significant, the magnitudes of the depolarization current and the real parts of the complex permittivity of these two oils are similar.

The time dependent curves of depolarization current of the mineral oil are well fitted using the exponential equations. The total charge density in the charge layer increases with temperature and ageing. The calculated ionic conductivity does not change much with the electric field. The total calculated conductivity increases with temperature and ageing. Even the values of the real part of the complex permittivity calculated using modified Coelho model is slightly higher than the experimental data, the tendency of the theoretical values and the experimental values are coherent.

This paper has proved that by introducing a new type of charge carriers, the high mobility charge carriers, the classic ionic drift and diffusion theory can be used to explain the electric conduction phenomena in mineral oil under both $\mathrm{AC}$ and DC field. Also, the polarization and depolarization current measurement can provide a brief view of the dielectric characteristics of charge carriers in mineral oil, which may be used in oil condition diagnostics in future. However, the polarization theory developed in this paper still needs improvement and more research is needed.

\section{ACKNOWLEDGMENT}

The authors are grateful for the financial support from National Grid UK.

\section{REFERENCES}

[1] I. Fofana, A. Bouaïcha, M. Farzaneh and J. Sabau, "Aging Behavior of Mineral Oil and Ester Liquids: A Comparative Study", IEEE Conf. Electr. Insul. Dielectr. Phenomena, Quebec, Canada, pp. 87-90, 2008.

[2] R. Sanghi, "Chemistry Behind the Life of a Transformer", Resonance, Vol. 8, pp. 17-23, 2003.

[3] T. K. Saha and P. Purkait, "Understanding the Impacts of Moisture and Thermal Ageing on Transformer's Insulation by Dielectric Response and Molecular Weight Measurements", IEEE Trans. Dielectr. Electr. Insul., Vol. 15, pp. 568-582, 2008.
[4] A. W. Stannett, "The Conductivity of Hydrocarbon Transformer Oil Containing Water and Solid Conducting Particles", British J. Appl. Phys., Vol. 2, pp. 110-114, 1951.

[5] M. Butcher, A. Neuber, M. D. Cevallos, J. C. Dickens and H. Krompholz, "Conduction and breakdown mechanismsin transformer oil", IEEE Trans. Plasma Sci., Vol. 34, pp. 467 -475, 2006.

[6] G. Jaffé, "Theorie der Leitfähigkeit polarisierbarer Medien. I", Ann. Phys., Vol. 408, pp. 217-248, 1933

[7] G Jaffé, "Theory of Conductivity of Semiconductors", Phys. Rev., vol. 85, pp. 354-363, 1952.

[8] H. C. Chang and G. Jaffé, "Polarization in Electrolytic Solutions. Part I. Theory”, J. Chem. Phys., Vol. 20, pp. 1071-1087, 1952.

[9] G. Jaffé and C. Z. LeMay, "On Polarization in Liquid Dielectrics", J. Chem. Phys., vol. 21, pp. 920-928, 1953.

[10] J. R. Macdonald, "Theory of ac Space-Charge Polarization Effects in Photoconductors, Semiconductors, and Electrolytes", Phys. Rev., Vol. 92, pp. 4-17, 1953.

[11] U. Gafvert, A. Jaksts, C. Tornkvist and L. Walfridson, "Electrical field distribution in transformer oil", IEEE Trans. Electr. Insul., Vol. 27, pp. 647-660, 1992.

[12] O. Hjortstam, J. Schiessling, Y. V. Serdyuk, S. M. Gubanski, "Measurements of ion mobility in transformer oil: Evaluation in terms of ion drift", IEEE Conf. Electr. Insul. Dielectr. Phenomena, Quebec, Canada, pp. 495-498, 2012.

[13] N. Lavesson, L. Walfridsson, O. Hjortstam and J. Schiessling, "Modelling and measurement of field dependent resistivity of transformer oil", IEEE Int'1. Conf. Dielectr. Liquids, pp. 1-4, 2014.

[14] Y. Zhou, M. Hao, G. Chen, G. Wilson and P. Jarman, "Study of the dielectric response in mineral oil using frequency-domain measurement", J. Appl. Phys., Vol. 115, 124105, 2014.

[15] D. G. Frood and T. J. Gallagher, "Space-Charge Dielectric Properties of Water and Aqueous Electrolytes", J. Mol. Liquids, Vol. 69, pp. 183-200, 1996.

[16] L. Onsager, "Deviations From Ohm's Law In Weak Electrolytes", J. Chem. Phys., Vol. 2, pp. 599-615, 1934

[17] F. Pontiga and A. Castellanos, "Electrical Conduction of Electrolyte Solutions in Nonpolar Liquids", IEEE Trans. Indust, Appl., Vol. 32, pp. $816-824,1996$.

[18] F. Pontiga and A. Castellanos, "The Effect of Field-Enhanced Injection and Dissociation on the Conduction of Highly-Insulating Liquids", IEEE Trans. Dielectr. Electr. Insul., Vol. 3, pp.792 -799, 1996.

[19] S. Patankar, Numerical Heat Transfer and Fluid Flow, Hemisphere Publishing Corporation, New York, 1980.

[20] I. Adamczewski, Ionization, Conductivity and Breakdown in Dielectric Liquids, Taylor and Francis, 1969.

[21] A. Küchler, M. Liebschner, A. Reumann, C. Krause, U. Piovan, B. Heinrich, R. Fritsche, J. Hoppe, A. Langens and J. Titze, "Evaluation of Conductivities and Dielectric Properties for Highly Stressed HVDC Insulating Materials", CIGRE Session 2010, Paper D1_106_2010, 2010.

[22] A. R. Nosseir, "Fluctuations of dc conduction in mineral oil under high electrical fields", IEEE Trans. Electr. Insul., Vol. 8, pp. 118-122, 1973.

[23] N. Felici, "DC Conduction in Liquid Dielectrics (Part I)", Dir. Current, Vol. 2, pp. $90-99,1972$.

[24] N. Felici, J. P. Gosse "Injection d'ions par des électrodes métalliques dans les hydrocarbures liquides de résistivité élevée", Rev. Phys. Appl., Vol. 14, pp. 629-633, 1979.

[25] A. Denat, B. Gosse and J. P. Gosse, "High Field DC and AC Conductivity of Electrolyte Solutions in Hydrocarbons", J. Electrostat., Vol. 11, pp. 179-194, 1982.

[26] A. Denat, B. Gosse and J. P. Gosse, "Ion Injections in Hydrocarbons", J. Electrostat., vol. 7, pp. 205-225, 1979.

[27] A. Alj, A. Denat, J. P. Gosse and B. Gosse, "Creation of Charge Carriers in Nonpolar Liquids", IEEE Trans. Electr. Insul., Vol. 20, pp. 221-231, 1985.

[28] R. Bartnikas, Engineering Dielectrics, Vol. III, Electrical Insulating Liquids, ASTM, 1994.

[29] P. A. Von Guggenberg, "Experimental Investigation of the Heterogeneity of the Double Layer in Transformer Oil", IEEE Conf. Electr. Insul. Dielectr. Phenomena, pp. 68-71, 1993.

[30] Y. Zhou, M. Hao, G. Chen, G. Wilson and P. Jarman, "A New Approach to Understanding the Frequency Response of Mineral Oil", IEEE Int'l. Conf. Dielectr. Liquids, Vol. 1, pp. 1-4, 2014. 


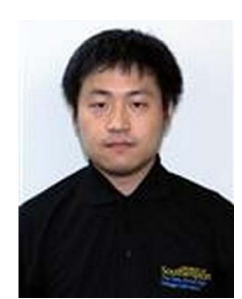

Yuan Zhou was born in China in 1987. He received his B.Eng. degree in electrical engineering from Xi'an Jiaotong University, China, in 2009. Since 2011, he has been a Ph.D. degree student in the University of Southampton, UK. His main research interests include electrical conduction and space charge in the oil impregnated pressboard.

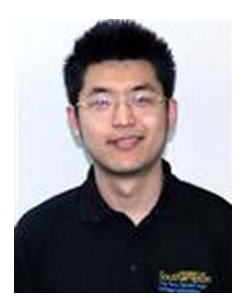

Miao Hao was born in China in 1987. He received his B.Eng. degree (2009) from Xi'an Jiaotong University, China and M.Sc. degree (2011) from University of Southampton, UK in electrical. Since 2011, he has been a Ph.D. student in the University of Southampton, UK. His main research interests include space charge and ageing mechanism in the oil impregnated pressboard insulation system for HVDC converter transformer.

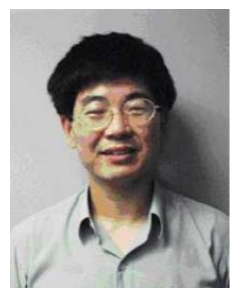

George Chen (SM'11) was born in China in 1961. He received the B.Eng. (1983) and M.Sc. (1986) degrees in electrical engineering from Xi'an Jiaotong University, China. After he obtained the Ph.D. degree (1990) from the University of Strathclyde, UK, on the work of permanent changes in electrical properties of irradiated low-density polyethylene, he joined the University of Southampton as postdoctoral research fellow and became a senior research fellow subsequently. In 1997 he was appointed as a research lecturer and promoted to a Reader in 2002. He is now the professor of high voltage engineering at the University of Southampton and a visiting professor of Xi'an Jiaotong University. Over the years, he has developed a wide range of interests in high voltage engineering and electrical properties of materials and published over 300 papers. He is active in the HVDC systems and involved with technical working groups in both IEEE and CIGRE.

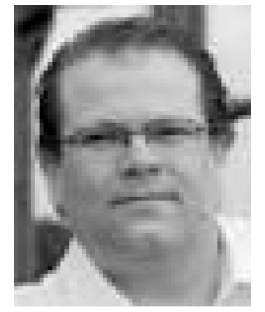

Gordon Wilson completed his chemistry degree at the University of Surrey in 1995, this was followed by a National Grid-sponsored Ph.D. degree in characterizing mineral transformer oil, also at the University of Surrey. He joined National Grid in 1999 as an oil chemist providing support to a team of transformer specialists. Since 2007 he has worked on transformer thermal ratings whilst retaining responsibility for transformer oil issues. Gordon is a member of the BSi National Committee for electrotechnical fluids, he is the UK regular member for CIGRE Study Committee on materials and he is a member of the IEEE Dielectrics and Electrical Insulation Society.

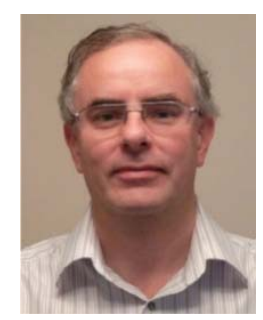

Paul Jarman was born in London on 27 September 1962, and graduated from Cambridge University in 1984 with an Honours degree in electrical science. He joined the Central Electricity Generating Board, Research Division, working on, amongst other projects, FRA testing of transformers. In 1990, he joined the National Grid as a transformer engineer, becoming head of transformers in 1998. Since 2001, Jarman has been National Grid's technical specialist for transformers now within the Asset Management group. Jarman is chairman of IEC TC14, the international committee for power transformer standards; is the UK regular member of CIGRE study committee A2 for transformers; and has recently been the convenor of a CIGRE group on transformer monitoring. He is a chartered electrical engineer and member of the IET. 